\title{
Scaling of the coercivity with the geometrical parameters in epitaxial Fe antidot arrays
}

\author{
E. Paz, ${ }^{1}$ F. Cebollada, ${ }^{2, a)}$ F. J. Palomares, ${ }^{1}$ J. M. González, ${ }^{3}$ M.-Y. Im, ${ }^{4}$ and P. Fischer ${ }^{4}$ \\ ${ }_{1}^{1}$ Instituto de Ciencia de Materiales de Madrid (CSIC), Cantoblanco, 28049 Madrid, Spain \\ ${ }^{2}$ POEMMA-CEMDATIC, ETSI de Telecomunicación, Universidad Politécnica de Madrid, 28040 Madrid, Spain \\ ${ }^{3}$ Unidad Asociada ICMM-IMA, Cantoblanco, 28049 Madrid, Spain \\ ${ }^{4}$ Center for X-ray Optics, Lawrence Berkeley National Laboratory, Berkeley, California 94720, USA
}

(Received 27 February 2012; accepted 5 March 2012; published online 10 April 2012)

\begin{abstract}
We studied a series of square lattice antidot arrays, with diameter and lattice parameter from hundreds of nanometers to some microns, fabricated using two lithography techniques in epitaxial $\mathrm{Fe}(001)$ films. The coercivity increase of each array with respect to its base film can be scaled to a simple geometric parameter, irrespective of the lithography technique employed. Magnetic transmission $\mathrm{x}$-ray microscopy studies, in arrays fabricated on polycrystalline Fe films deposited on silicon nitride membranes, evidenced the propagation of reversed domains from the edges of the arrays, in agreement with the coercivity analysis of the epitaxial arrays and with micromagnetic models. (C) 2012 American Institute of Physics. [http://dx.doi.org/10.1063/1.3702584]
\end{abstract}

\section{INTRODUCTION}

Patterned magnetic thin films have attracted a lot of interest due to the possibility of tailoring their magnetization processes through their geometrical structure and also to their potential for technological applications, e.g., in the field of magnetic storage, sensors, radio frequency components or magnonic crystals. ${ }^{1,2}$ Antidot arrays, which consist of an ordered structure of holes in an otherwise continuous film, produce dramatic variations of the hysteretic parameters: additional contributions to the anisotropy give rise to novel domain structures and modified reversal mechanisms, resulting in an increase of the switching fields with respect to those of the continuous films. ${ }^{2-6}$

The hysteresis phenomena occurring in antidot arrays are strongly influenced by inhomogeneous magnetization structures generated around the antidots to reduce the dipolar energy. For a given set of exchange, magnetization and anisotropy constants, resulting in exchange and magnetostatic correlation lengths smaller than the antidots dimensions, different regimes can be considered, depending on the size and separation of the antidots. In the so called diluted regime -when the distance between the antidots is many times their diameter - the coercivity can be treated in the frame of conventional strong pinning models. ${ }^{7}$ On the contrary, in the concentrated regime, when the size of the inhomogeneous structures generated due to the dipolar energy is comparable to the dimensions of the array unit cell, the minimization of the energy around the antidots leads to the stabilization of the global magnetization. The large increase in dipolar energy required to modify those inhomogeneities is responsible for the increase of the switching field, which is a general trend in all arrays. Another common feature of antidot arrays is related to the symmetry of the coercivity and the remanence. In most cases the angular dependence of the both parameters is

\footnotetext{
a) Author to whom correspondence should be addressed. Electronic mail: fcebollada@etsit.upm.es.
}

dictated by the symmetry of the array, ${ }^{5,8,9}$ with easy (hard) axis corresponding to the direction along which the distance between the antidots is maximum (minimum), although the quality and shape of the antidot edges has also a strong influence. ${ }^{8,10}$ Regarding the magnetization reversal mechanisms, both simulations and experimental works have evidenced that the reversal takes place through the propagation of reversed domains probably nucleated in the continuous films surrounding the arrays. The specific features of the propagation scheme depend on the geometry of the array and anisotropy contributions other than just that due to the symmetry of the array. ${ }^{11,12}$

Although much effort has been devoted to study the influence of the array symmetry on the hysteresis, little work has been carried out on the variation of the coercivity with the antidot sizes and distances for a given geometry. Just some simulations and a few experimental results suggest that the coercivity tends to increase with decreasing distance between the antidots. ${ }^{13,14}$ In this work we present a systematic study of the coercivity of a large series of square lattice arrays, with antidots diameters between $300 \mathrm{~nm}$ and $1 \mu \mathrm{m}$ and distance between their edges from $200 \mathrm{~nm}$ to $2 \mu \mathrm{m}$, fabricated on epitaxial $\mathrm{Au}(001) / \mathrm{Fe}(001) / \mathrm{Mg}(001)$ films by using two patterning techniques.

\section{SAMPLES AND EXPERIMENTAL}

Fe (001) films were deposited at room temperature on $\mathrm{MgO}(001)$ substrates, previously annealed for water desorption for $25 \mathrm{~min}$ at $200{ }^{\circ} \mathrm{C}$, by pulsed laser deposition, using a Nd-YAG laser ( $\lambda=532 \mathrm{~nm}, 4 \mathrm{~ns}$ pulses, $25 \mathrm{~mJ} /$ pulse $)$. After deposition the films were annealed at $400{ }^{\circ} \mathrm{C}$ in order to improve their crystalline quality and surface roughness, and then a $\mathrm{Au}$ (001) capping layer, $3 \mathrm{~nm}$ thick, was deposited by molecular beam epitaxy for protection purposes. The whole process was carried out under ultrahigh vacuum conditions. A detailed structural and morphological analysis evidenced that the films are highly epitaxial, with a $\mathrm{Au}(001)[100] /$ $\mathrm{Fe}(001)[100] / / \mathrm{MgO}(001)[110]$ relation. $^{15}$ 
TABLE I. Diameter $(D)$ and separation between the antidot edges $(\lambda)$. The six EBL arrays were fabricated in the same film; arrays marked FIB1 and FIB2 were fabricated in two different films.

\begin{tabular}{lcccccc}
\hline \hline$\lambda(\mathrm{nm})$ & 200 & 300 & 400 & 500 & 750 & 1000 \\
\hline EBL & $D=400 \mathrm{~nm}$ & $\begin{array}{l}D=300 \mathrm{~nm} \\
D=400 \mathrm{~nm}\end{array}$ & $D=400 \mathrm{~nm}$ & & $D=750 \mathrm{~nm}$ & $D=1000 \mathrm{~nm}$ \\
FIB & & & $D=500 \mathrm{~nm}(\mathrm{FIB} 2)$ & & $D=1000 \mathrm{~nm}(\mathrm{FIB} 2)$ & $D=1000 \mathrm{~nm}(\mathrm{FIB} 1)$ \\
\hline \hline
\end{tabular}

A total of 9 square lattice antidot arrays, with diameter $D$ between $300 \mathrm{~nm}$ and $1 \mu \mathrm{m}$ and separation between their edges $\lambda$ from $200 \mathrm{~nm}$ to $2 \mu \mathrm{m}$, were fabricated in three different Fe films using focused ion beam (FIB) and electron beam lithography (EBL) (see Table I and Fig. 1). FIB lithographed arrays, $500 \times 460 \mu \mathrm{m}^{2}$, were produced by bombardment with a $100 \mathrm{pA}, \mathrm{Ga}^{+}$beam accelerated at $30 \mathrm{kV}$, using a FEI Strata DB23 at the Nanotechnology Platform of the Barcelona Science Park. A frame was carved around these arrays to isolate them from the surrounding $\mathrm{Fe}$ film. The EBL arrays, $1 \times 1 \mathrm{~mm}^{2}$, were fabricated using positive resist and were processed by means of a Raith e-LiNE at the Nano-Bio Center of the Technical University of Kaiserslautern. A $200 \mathrm{~nm}$ thick $950 \mathrm{~K}$ PMMA layer was deposited and then exposed by a $20 \mathrm{kV}, 0.15 \mathrm{nA}$ e-beam, yielding a $200 \mu \mathrm{C}$ $\mathrm{cm}^{-2}$ dose. After the developing and subsequent lift-off process an $\mathrm{Ar}^{+}$ion etching was carried out using an IBE-RIBE, Roth \& Rau IonSys 500. All the arrays were oriented with their diagonals parallel to the [100] and [010] directions, i.e., coincident with the $\mathrm{Fe}$ easy magnetocrystalline directions (Fig. 1).

The magnetic characterization of the arrays was carried out at room temperature by means of a vectorial magnetooptic Kerr effect device (MOKE) under maximum applied fields of $5 \mathrm{kOe}$. In order to gain insight into their microscopic magnetization mechanisms, an independent set of arrays was studied by magnetic transmission soft $\mathrm{x}$-ray microscopy (MTXM) at beamline 6.1.2 at the advanced light source

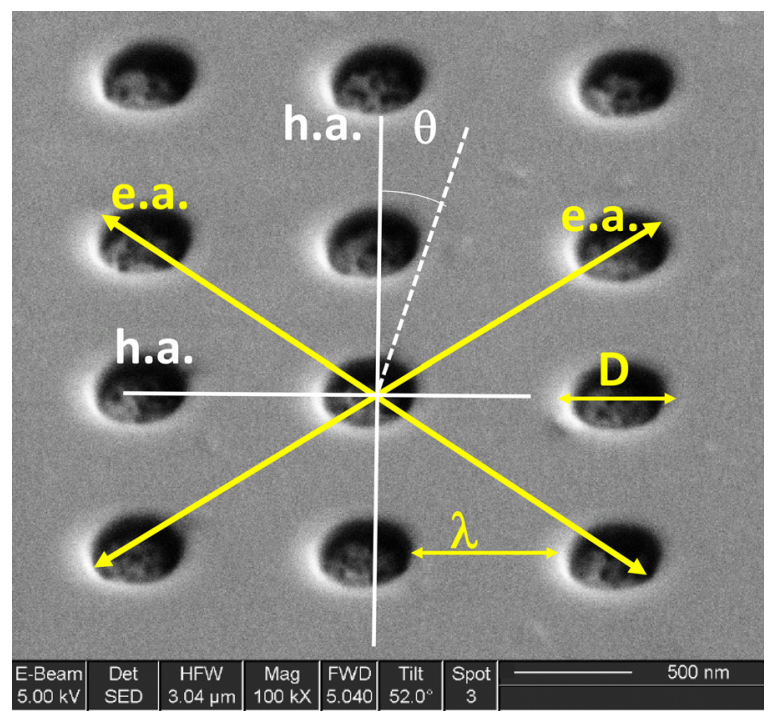

FIG. 1. SEM image of an array fabricated by FIB with $D=\lambda=500 \mathrm{~nm}$ showing the orientation of the magnetocrystalline easy and in-plane hard axes (e.a. and h.a., respectively).
(ALS) in Berkeley, CA. These arrays were fabricated by FIB, with a $300 \mathrm{pA}$ intensity beam, on $20 \mathrm{~nm}$ thin Fe films deposited on $200 \mathrm{~nm}$ thick silicon nitride membranes by means of the procedure already outlined. Contrary to the films deposited on $\mathrm{MgO}$, the use of silicon nitride membranes, which is compulsory to study samples in transmission geometry, gives rise to the generation of polycrystalline Fe films. The high spatial resolution of MTXM, of the order of $20 \mathrm{~nm}$, and the possibility to acquire images under magnetic applied field allows the observation of fine details of the magnetization configurations and of the reversal behavior of the arrays. ${ }^{11,16}$ The magnetic contrast results from x-ray magnetic circular dichroism (XMCD). Two images taken with both light polarizations (circular left and right) were recorded and divided by each other. Since XMCD scales with the projection of the local magnetization onto the photon propagation direction, the sample was tilted with its normal forming an angle of $30^{\circ}$ with respect to photon transmission direction and therefore we are sensitive to the in-plane magnetization/domains.

\section{RESULTS AND DISCUSSION}

Figure 2 shows the hysteresis loops of an array with $D=1 \mu \mathrm{m}$ and $\lambda=2 \mu \mathrm{m}$ and of the continuous film on which it was lithographed, measured with the field applied at an angle $\theta=15^{\circ}$ (depicted in Fig. 1) with respect to one of the in-plane hard magnetocrystalline axes (the in-plane hard magnetocrystalline axes will be simply referred to as hard magnetocrystalline axes in the paper). The loop corresponding to the film has two switching fields $H_{S 1}$ and $H_{S 2}$, at about 25 and $65 \mathrm{Oe}$, respectively, whereas the switching fields of

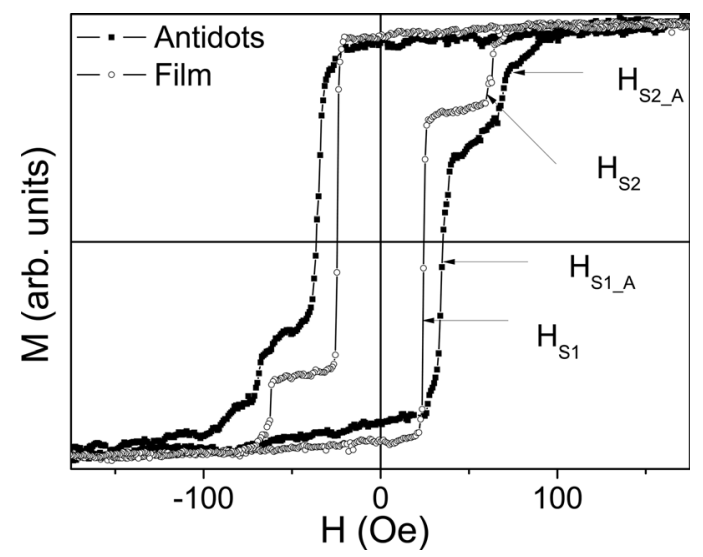

FIG. 2. Hysteresis loops, measured with the field applied at an angle $\theta=15^{\circ}$ with respect to the hard axis, corresponding to the array with $D=1 \mu \mathrm{m}$ and $\lambda=2 \mu \mathrm{m}$ (FIB2) and to the continuous film in which it was fabricated. 
the array $H_{S 1 \_A}$ and $H_{S 2 \_A}$ are slightly above those of the film, near 35 and $70 \mathrm{Oe}$, respectively. The coincidence of the magnetostatic easy and hard directions of the array (diagonal and side of the square lattice, respectively) with the magnetocrystalline easy and hard axes, also respectively, gives rise to a purely biaxial system. As is well known, the presence of an intermediate energy minimum during the magnetization reversal in thin films with four-fold in-plane anisotropy might give rise to hysteresis loops characterized by either one or two magnetization jumps, depending on the angle between the applied field and the easy axes. ${ }^{17,18}$ Although not shown, the angular dependence of the switching fields of the arrays showed a good agreement with previously reported results, ${ }^{12}$ which evidenced the existence of two switching fields which converge to a single value when the field is applied along the easy axis and that the second magnetization jump becomes negligible when it is applied along the hard axis. Different models have been analyzed by means of micromagnetic simulations to understand the magnetization mechanisms of antidot arrays. A first approach consists in considering that the reversal takes place fully inside the array, proceeding from the magnetization inhomogeneities around the antidots. A second approach is based on the nucleation of the demagnetization process in the continuous film surrounding the array, at lower fields, and its subsequent propagation inside the array. Although both models provide two magnetization jumps, with an intermediate minimal energy direction, the first model results in a very weak angular dependence of the switching fields. In contrast, the second model shows a good agreement with the experimentally observed behavior. ${ }^{12}$ According to it, the domain generated outside the array reaches its border and then it triggers the switching of the array to an intermediate easy direction. The array magnetization gets stabilized around the antidots and, after the full reversal of the external magnetization, a wall is formed at the border. The depinning of this wall from the outer lines of antidots leads to the complete reversal of the array. Consequently, the switching fields calculated for the array take place at higher values than those corresponding to the film.

Assuming that the magnetization reversal is externally nucleated, a certain dependence of the switching fields of each array on the specific switching fields value of its corresponding continuous film could be expected. As previously mentioned, the arrays were fabricated on three different films, which had different switching fields even though they were deposited under highly controlled conditions. The switching field (coercivity) values measured with the applied field along the easy (hard) axes are, approximately, $48 \mathrm{Oe}$ (40 Oe) for the film with the EBL arrays, 30 Oe (22 Oe) for the film containing the FIB2 arrays and $11 \mathrm{Oe}(9 \mathrm{Oe})$ for the film with the FIB1 array. The coercivity of the arrays shows a general tendency to increase with decreasing separation between the antidots $\lambda$, although with a certain dispersion dependent on the continuous film on which each array was fabricated. An important issue is related to the increase in coercivity of the arrays $\Delta H_{C}$, along both the easy and hard axis, with respect to the values measured in their corresponding films (Fig. 3). The global behavior fits reasonably a sin-

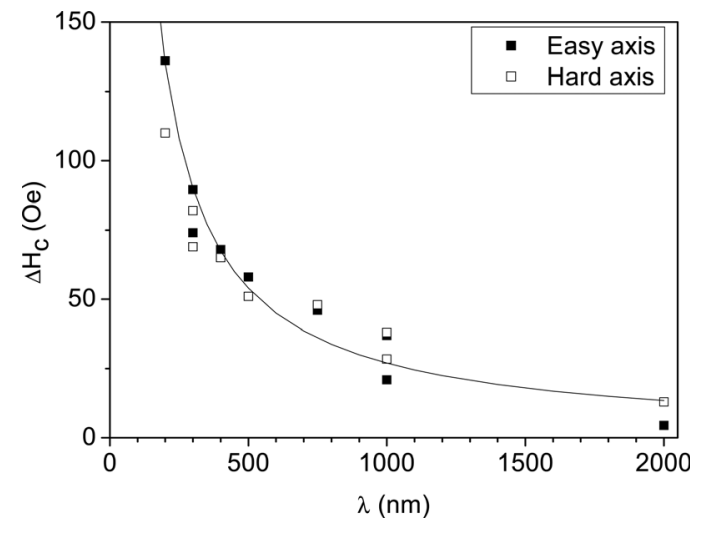

FIG. 3. Increase of the coercivity of all arrays with respect to that of the continuous films on which each one was fabricated, along the easy and hard axes, as a function of the separation between the antidots $\lambda$.

gle $1 / \lambda$ line, irrespective of the lithography technique employed to fabricate the arrays. When plotted as a function of the diameter $D$, the coercivity also increases with decreasing $D$, although in this case with a very large dispersion which allows no significant scaling. Although some authors have proposed a tentative $\lambda$ scaling of the coercivity for antidots, both simulations and experimental data are not conclusive. ${ }^{13,19}$ In fact, any physically meaning parameter scaling the coercivity must involve, in some way, both the separation $\lambda$ and the diameter $D$. Taking into account that one of the key issues of the magnetization processes of antidot arrays lies in the formation of highly stable, inhomogenous magnetization structures ("pinned regions") generated around the antidots, we have considered their relative area (with respect to the total Fe area of the array) as a scaling parameter. The characteristic length of magnetization inhomogeneities in iron is of the order of the domain wall width $\delta$, about $50 \mathrm{~nm}$ for $\mathrm{Fe},{ }^{20}$ from which the relative size $S$ of the pinned regions (inset in Fig. 4) can be estimated as

$$
S=\frac{\pi\left[(D+\delta)^{2}-D^{2}\right]}{4(D+\lambda)^{2}-\pi D^{2}}
$$

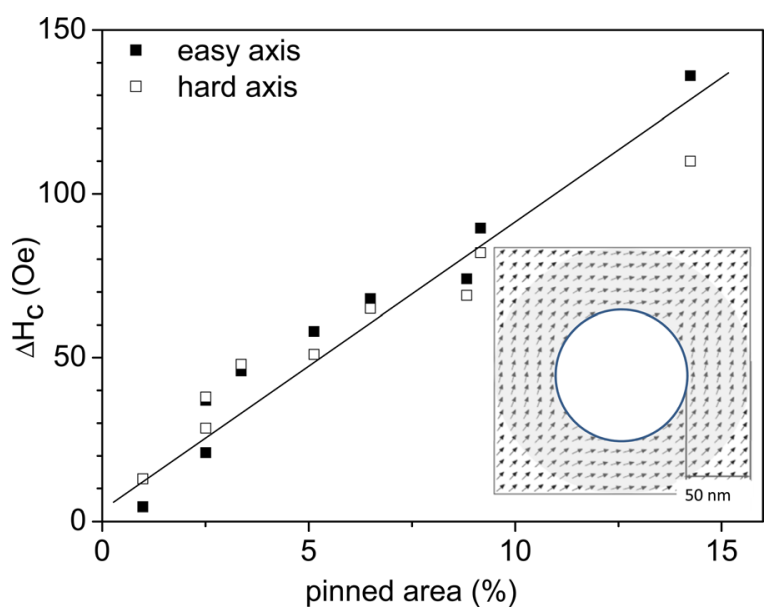

FIG. 4. Increase of the coercivity of the arrays as a function of the area percentage of the "pinned" regions calculated from $S$ in Eq. (1). Inset: scheme of the "pinned region" around an antidot. 


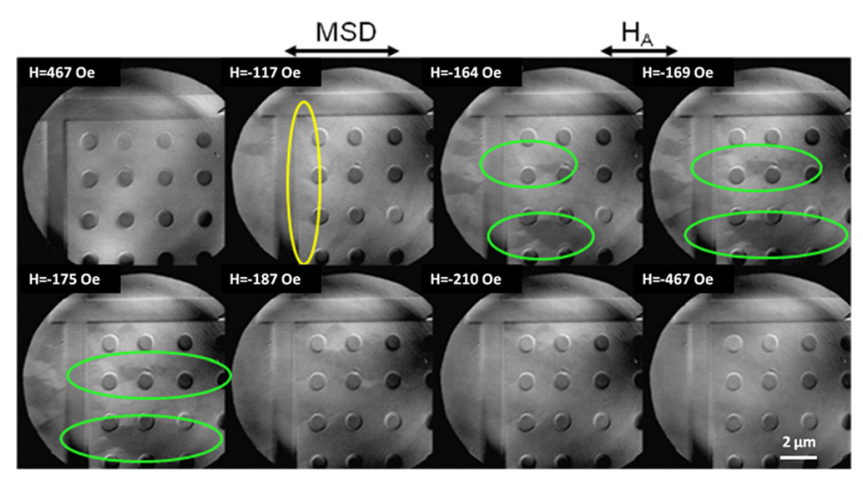

FIG. 5. Sequence of the magnetization reversal starting from the edges of an array with $\mathrm{D}=\lambda=1 \mu \mathrm{m}$, studied by MTXM.

The increase in coercivity $\Delta H_{C}$ exhibits a fairly good linear scaling with area percentage of the "pinned regions," including both the easy and hard axis values in the same line. This confirms the fundamental role that the stabilization of the magnetization around the antidots plays in the switching mechanisms, It is important to remark that although the anisotropy of iron is relatively high, $k \sim 4.8 \cdot 10^{5} \mathrm{Jm}^{-3}$, the dipolar-to-anisotropy energy ratio, $\mu_{\mathrm{o}} M_{S}{ }^{2} / k$, is still very high, of the order of $10^{2}$, due to its high saturation magnetization $M_{S}$. This explains the strong dependence of the coercivity on the dimensions of the morphological features of the arrays in spite of the well defined single crystalline character of the samples.

We present in Fig. 5 a series of MTXM images corresponding to the corner of an array with $D=\lambda=1 \mu \mathrm{m}$ (fabricated in one of the polycrystalline Fe films deposited on a silicon nitride membrane) obtained by sweeping the in-plane magnetic field $H_{A}$ from 470 to $-470 \mathrm{Oe}$, approximately, which is enough to drive the magnetization from positive to negative saturation. After positive saturation the field is reversed and, as can be seen, upon the application of a negative field of $-117 \mathrm{Oe}$, approximately, dark traces appear between the left edge of the array and the first antidot column and also along some of the diagonals between the antidots of the first and second columns. At -164 Oe the contrast is enhanced and large dark, reversed regions appear connected to the edge of the array which propagate to the right at about -169 Oe. At -186 Oe the magnetization is almost fully reversed except for just some regions, mainly between the upper row and the edge of the array and, finally, the full reversal is accomplished between -210 and -470 Oe. Further image sequences with identical field runs, although not strictly repetitive, evidenced the same features: the presence of dark traces near the left edge which propagate to the right along the rows, at applied fields between -160 and -170 Oe, approximately, with just some regions that remain unswitched near the upper edge. Although the MTXM images in polycrystalline samples cannot be taken as strictly conclusive with respect to the actual reversal mechanism of the single crystalline epitaxial arrays, the fact that the dipolar-to anisotropy energy ratio in $\mathrm{Fe}$ is rather large, of the order of $10^{2}$, allows to assume that the reversal mechanisms is not going to be dramatically modified in our epitaxial arrays. The propagation of reversed domains along the rows parallel to the applied field seems to be a general trend that has also been observed in arrays of Co antidots ${ }^{6,11}$ and it is consistent with the predictions of several models — carried out under the assumption of single crystalline Fe arrays with identical orientation of the easy magnetocrystalline axes (i.e., along the diagonals of the arrays)—which essentially indicate that the magnetization reversal proceeds by means of a nucleation-propagation sequence generated in the continuous film surrounding the arrays. ${ }^{12}$

\section{CONCLUSIONS}

As a conclusion, we have studied the magnetization reversal mechanism and the dependence of the coercivity on the morphological parameters of a large series of antidot arrays fabricated on single crystalline Fe films using two different lithography techniques. We have shown that the coercivity increases with decreasing separation between the antidots although it also depends on the antidot diameter and on the specific coercivity value of the continuous films on which each array was fabricated. The increase in coercivity with respect to that of the films can be reasonably fitted to a $1 / \lambda$ law for all arrays, irrespective of the lithography technique employed. To take simultaneously into account the diameter and separation between the antidots a parameter involving both of them, the area percentage of the Fe regions "pinned" around the antidots, was used, which revealed a fairly good linear scaling. The specific analysis of the reversal mechanism, based on the study of the angular dependence of the hysteresis parameters and on the visualization of the reversal processes in polycrystalline arrays by MTXM, suggests that the magnetization reversal proceeds from the edges of the arrays, in agreement with the results of previous micromagnetic simulations and with the observed dependence of the coercivity of each array on that on its corresponding continuous film.

\section{ACKNOWLEDGMENTS}

This work has been carried out under the financial support of the Spanish MICINN (grants MAT2007-66719-C03, MAT2010-18432 and FUNCOAT Consolider CSD200800023). The operation of the microscope at the Advanced Light Source was supported by the Director, Office of Science, Office of Basic Energy Sciences, of the U.S. Department of Energy under Contract No. DE-AC02-05CH11231. The authors also wish to thank the Nano-Bio Center of the Technical University of Kaiserslautern and the Nanotechnology Platform of the Barcelona Science Park for technical support.

${ }^{1}$ B. D. Terris and T. Thomson, J. Phys. D 38, R199 (2005).

${ }^{2}$ S. Tacchi, M. Madami, G. Gubbiotti, G. Carlotti, A. O. Adeyeye, S. Neusser, B. Botters, and D. Grundler, IEEE Trans. Magn. 46, 1440 (2010).

${ }^{3}$ I. Guedes, M. Grimsditch, V. Metlushko, P. Vavassori, R. Camley, B. Ilic, P. Neuzil, and R. Kumar, Phys. Rev. B 66, 014434 (2002).

${ }^{4}$ U. Welp, V. K. Vlasko-Vlasov, G. W. Crabtree, C. Thompson, V. Metlushko, and B. Ilic, Appl. Phys. Lett. 79, 1315 (2001).

${ }^{5}$ P. Vavassori, G. Gubbiotti, G. Zangari, C. T. Yu, H. Yin, H. Jiang, and G. J. Mankeyd, J. Magn. Magn. Mater. 242-245, 585 (2002).

${ }^{6}$ L. J. Heyderman, F. Nolting, D. Backes, S. Czekaj, L. Lopez-Diaz, M. Kläui, U. Rüdiger, C. A. F. Vaz, J. A. C. Bland, R. J. Matelon, U. G. Volkmann, and P. Fischer, Phys. Rev. B 73, 214429 (2006).

${ }^{7}$ A. Pérez-Junquera, J. I. Martín, M. Vélez, J. M. Alameda, J. V. Anguita, F. Briones, E. M. González, and J. L. Vicent, Nanotechnology 15, S131 (2004). 
${ }^{8}$ N. G. Deshpande, M. S. Seo, X. R. Jin, S. J. Lee, Y. P. Lee, J. Y. Rhee, and K. W. Kim, Appl. Phys. Lett. 96, 122503 (2010).

${ }^{9}$ F. Pigazo, F. García Sánchez, F. J. Palomares, J. M. González, O. Chubykalo-Fesenko, F. Cebollada, J. M. Torres, J. Bartolomé, and L. M. García Vinuesa, J. Appl. Phys. 99, 08S503 (2006).

${ }^{10}$ C. C. Wang, A. O. Adeyeye, and N. Singh, Nanotechnology 17, 1629 (2006).

${ }^{11}$ L. J. Heyderman, S. Czekaj, F. Nolting, D. -H. Kim, and P. Fischer, J. Magn. Magn. Mater. 316, 99 (2007).

${ }^{12}$ F. García-Sánchez, E. Paz, F. Pigazo, O. Chubykalo-Fesenko, F. J. Palomares, J. M. González, F. Cebollada, J. Bartolomé, and L. M. García, Europhys. Lett. 84, 67002 (2008).

${ }^{13}$ I. Ruiz-Feal, L. Lopez-Diaz, A. Hirohata, J. Rothman, C. M. Guertler, J. A. C. Bland, L. M. Garcia, J. M. Torres, J. Bartolome, F. Bartolome, M. Natali, D. Decanini, and Y. Chen, J. Magn. Magn. Mater. 242-245, 597 (2002).
${ }^{14}$ J. M. González, O. Chubykalo-Fesenko, F. García-Sánchez, J. M. TorresBruna, J. Bartolomé, and L. M. García Vinuesa, IEEE Trans. Magn. 41, 3106 (2005)

${ }^{15}$ E. Paz, F. Cebollada, F. J. Palomares, F. García-Sánchez, and J. M. González, Nanotechnology 21, 255301 (2010).

${ }^{16}$ P. Fischer, D. H. Kim, W. Chao, J. A. Liddle, E. H. Anderson, and D. T. Attwood, Mater. Today 9, 26 (2006).

${ }^{17}$ F. Cebollada, A. Hernando-Mañeru, A. Hernando, C. Martínez-Boubeta, A. Cebollada, and J. M. González, Phys. Rev. B 66, 174410 (2002).

${ }^{18}$ U. Ebels, A. O. Adeyeye, M. Gester, R. P. Cowburn, C. Daboo, and J. A. C. Bland, Phys. Rev. B 56, 5443 (1997).

${ }^{19}$ J. M. Torres-Bruna, J. Bartolomé, L. M. García-Vinuesa, F. GarcíaSánchez, J. M. González, and O. A. Chubykalo-Fesenko, J. Magn. Magn. Mater. 290-291, 149 (2005).

${ }^{20}$ S. Chikazumi, Physics of Magnetism (Krieger Publishing Co., Malabar, FL, 1978), p. 186. 
Journal of Applied Physics is copyrighted by the American Institute of Physics (AIP). Redistribution of journal material is subject to the AIP online journal license and/or AIP copyright. For more information, see http://ojps.aip.org/japo/japcr/jsp 